\title{
Sinus venous thrombosis: a rare complication of varicella zoster virus
}

\author{
Authors: Emadullah Raidullah ${ }^{\mathrm{A}}$ and Waqar $\mathrm{Gaba}^{\mathrm{A}}$
}

\section{Introduction}

Varicella zoster virus (VZV), a ubiquitous deoxyribonucleic acid (DNA) virus, is one of eight known human herpesviruses. Primary infection occurs via aerosols from skin vesicles from an infected person with varicella or zoster, resulting in the characteristic disseminated rash of varicella. VZV is also known to cause vasculopathy. It is associated with productive viral infection in arteries, as evidenced by the presence of multinucleated giant cells, herpesvirus particles, VZV DNA and VZV antigen in arteries. ${ }^{1}$ Autoantibodies to phospholipids and coagulation proteins (during or after varicella) may play a role in the occlusion of cerebral arteries. ${ }^{2}$

\section{Case presentation}

We present a case of a 36-year-old man who developed extensive cerebral venous sinus thrombosis following primary VZV infection. The patient present to the emergency department (ED) with a 12-hour history of upper and lower limb weakness which was associated with severe headache. There was no swallowing difficulty or vision changes. However, the patient reported that he had chickenpox 1 week prior, for which he used topical lotion. On examination, the patient had a muscle power score of 1 out of 5 in his left upper and lower limb; the rest of the neurological examination was normal. He was also found to have some fresh lesions and some crusted lesions over trunk, upper and lower limbs. An immediate computed tomography (CT) with contrast was carried out, which showed filling defect in the superior sagittal sinus confluence, both transverse and left sigmoid sinuses consistent with dural venous sinus thrombosis (Fig 1). Neurology and infectious disease consultations were carried out. The patient was started on intravenous acyclovir and therapeutic dose subcutaneous enoxaparin. Magnetic resonance imaging (MRI) was undertaken the following day, which confirmed the same findings of dural venous sinus thrombosis (Fig 2). On day 3 of hospitalisation, the patient started to regain power in his left upper and lower limb. He was moved to the rehabilitation ward where he received intensive rehabilitation. After 2 weeks the patient was discharged with normal power in his left upper and lower limb. We recommended subcutaneous enoxaparin for a total of 6 months.

\section{Conclusion}

We report on this case to raise awareness among clinicians so that this rare complication of primary VZV can be recognised

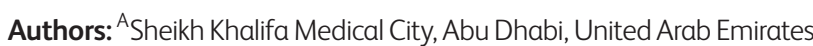

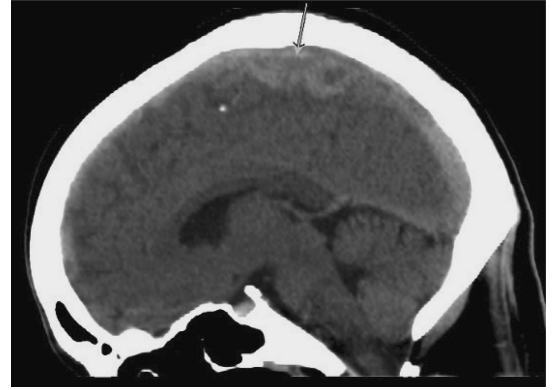

Fig 1. Computed tomography of the head (arrow showing dural sinus thrombosis).

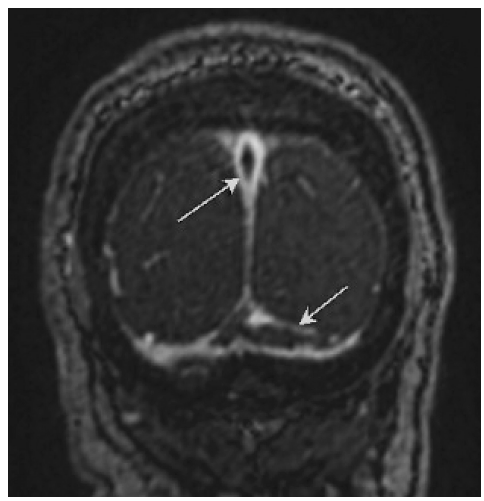

Fig 2. Magnetic resonance imaging of the head (white arrows showing dural sinus thrombosis).

in a timely manner and managed immediately, as this can be lifesaving.

\section{Conflicts of interest}

None declared.

\section{References}

1 Gilden DH, Kleinschmidt-DeMasters BK, Wellish M et al. Varicella zoster virus, a cause of waxing and waning vasculitis: the New England Journal of Medicine case 5-1995 revisited. Neurology 1996;47:1441-6.

2 Josephson C, Nuss R, Jacobson L et al. The varicella-autoantibody syndrome. Pediatr Res 2001;50:345-52. 